\title{
Psychosocial characteristics and HIV-related risky sexual behaviors among cisgender, transgender, and gender non- conforming MSM in China
}

\author{
Zhizhou Duan \\ Wuhan University \\ Liyin Wang \\ Wuhan University \\ Menglan Guo \\ wuhan university \\ Changmian Ding \\ Wuhan University \\ Danqin Huang \\ Wuhan University \\ Hong Yan ( $\square$ yanhmjxr@whu.edu.cn ) \\ Amanda Wilson \\ De Montfort University \\ Shiyue Li \\ Wuhan University
}

\section{Research article}

Keywords: Men who have sex with men, cisgender, transgender, gender non-conforming, psychosocial characteristics, HIV-related risky sexual behaviors

Posted Date: January 13th, 2021

DOI: https://doi.org/10.21203/rs.3.rs-51858/v2

License: (1) This work is licensed under a Creative Commons Attribution 4.0 International License. Read Full License

Version of Record: A version of this preprint was published at BMC Psychiatry on April 17th, 2021. See the published version at https://doi.org/10.1186/s12888-021-03189-z. 


\section{Abstract}

Background: While a growing number of studies focus on men who have sex with men (MSM), they typically ignore the heterogeneity of gender minorities within the MSM population. The recognition of new sub-groups among gender minorities (i.e., transgender and gender non-conforming), who also identify as MSM, play a considerable role in new HIV infections in China. Information on the psychosocial factors and HIV-related risky sexual behaviors requires further consideration to understand the prevalence of HIV infection among MSM within these gender minority sub-groups.

Methods: From September 2017 to January 2018, MSM were recruited in Wuhan, Nanchang, and Changsha cities in China. Participants were asked to fill out a structured self-administered questionnaire to assess depression, perceived social support, resilience, identity concealment, and HIV-related risky sexual behaviors.

Results: A total of 715 MSM completed the structured questionnaire, the number of gender minorities identifying as MSM were 63 and accounted for $8.8 \%$ of the population. Compared to the cisgender MSM population, transgender MSM were more likely to have a one-night stand/occasional partners ( $\mathrm{AOR}=3.49,95 \% \mathrm{Cl}=1.02-11.98)$, to have sex after drug use in the past six months $(A O R=2.57,95 \% \mathrm{Cl}=1.05-6.29)$, and to have reported a significantly lower likelihood of identity concealment (mean difference=-3.30, $95 \% \mathrm{Cl}=-5.86,-0.74, \mathrm{P}=0.01)$.

Conclusions: The findings highlight the significance of providing targeted interventions for different gender minorities within the MSM population. Research is required to further understand the relationship between gender identity, psychosocial characteristics, and HIV-related risky sexual behaviors.

\section{Background}

Gender minorities refer to those whose gender identity is not same as their birth-assigned sex (transgender) and those whose gender identity is not defined by the binary categories of women/men (gender non-conforming) [1, 2]. In recent studies around the world, there has been a call for further analysis of HIV interventions that target different gender identities among men who have sex with men (MSM) [3-5]. Previous research has shown that transgender and gender non-conforming MSM account for over $23 \%$ of the MSM population in Peru [6]. Jobson et al. found that $9 \%$ of individuals were identified as gender minorities within the MSM population in a city of South Africa [7].

Individual MSM identified as a gender minority report a higher likelihood of HIV risks in comparison with cisgender MSM [8, 9]. For example, compared to the cisgender MSM population, transgender MSM are at a higher risks of new HIV infections due to sociodemographic variables, having higher rates of substance use, and due to the higher engagement in sex work [10]. In a systematic review of MSM in low and middle income countries, transgender MSM had a higher prevalence of HIV and self-reported that they were more likely to engage in receptive anal intercourse without use of a condom $[9,11]$. A recent survey in Shanghai also documented that transgender and gender non-conforming MSM bore a higher HIV burden, with the prevalence of HIV-infection reaching as high as $12.4 \%$ [12].

In addition, a previous study conducted in China has suggested that transgender and gender non-conforming populations selfreport a higher incident of being bullied, higher experiences of neglect and abuse, and more depressive symptoms [13]. And the studies on transgender conducted in USA have found that psychosocial factors can increase the risk of HIV infection [14, 15]. Also, psychosocial factors including depression and stigma have been reported to increase the likelihood of HIV affecting in South African MSM [16]. So, psychosocial characteristics of gender minorities can't be ignored.

To date, the associated sub-groups within the MSM population, transgender and gender non-conforming, are not considered separate sub-groups within the MSM population in China [12]. Previous studies typically treat the MSM population as homogenous group and corresponding intervention measures have been generalized to target the MSM population as a whole [17], providing limited information on the impact of new HIV infections and transmission among MSM gender minorities [12, 18, 19]. In order to be effective in reducing HIV infections, growing evidence has suggested that it is important to create targeted intervention based on specific gender identity $[7,12]$. 
Therefore, the present study aims to explore the relationship between gender identity, psychosocial factors, and HIV related risky sexual behaviors to provide tailored interventions that can be more effective in reducing HIV infections in Chinese sexual minorities. First, this study identified the prevalence of transgender and gender non-conforming individuals within the MSM population. Second, the study assessed the HIV-related risky sexual behaviors and psychosocial characteristics of each subgroup of gender minority MSM, in order to provide new evidence for targeted interventions among the different MSM gender minority populations.

\section{Methods}

\subsection{Participants}

Between September 2017 and January 2018, a baseline survey of a four-year cohort study was conducted in three provincial capitals of China: Wuhan, Nanchang, and Changsha. The cohort study aims to examine the effect mechanisms of perceived stigma on risk behaviors, psychology and HIV infection among Chinese MSM. Participants were recruited through several ways (routine HIV testing services, peer recommendation, and outreach activities), with the help of the local Centers for Disease Control and Prevention (CDC), and MSM organizations (Tongxing organization, Xingyuan organization and Qingcai organization). The inclusion criteria for the study were: (1) they were 16 years or older; (2) they had experience of having sex with men; (3) they were sexually active during the past 6 months; (4) they self-reported no HIV infection; (5) they provided written informed consent. The participants were asked to complete the electronic questionnaire on tablets (e.g. iPads) on their own in a quiet room in order to protect the individual privacy, and the trained students or volunteers were available to provide clarity if participants were unsure what the question was asking. All participants who finished the survey received 50 RMB (approximately US \$7) as compensation for their time in this baseline survey. This study was approved by the Medical Ethics Committee of Wuhan University, China.

\subsection{Data Collection}

Socio-demographic Characteristics

Socio-demographic information included: age, ethnicity (Han, others), current education level (high school or lower, college, graduate or higher), current employment (employed, unemployed), current marital status (unmarried, married, divorced), and monthly income (<1000 Yuan, 1000-3000 Yuan, 3001-6000 Yuan, >6000 Yuan). Sexual orientation was also measured by a single item question, "what's your sexual orientation?", and participants could choose from four options, "gay", "bisexual”, "heterosexual”, and "unsure".

Gender identity

Gender identity (the primary "exposure" variable for the analysis) was measured by a single item, in order to identify transgender individuals, the question was asked "Do you agree with your sex assigned at birth?", with three options included: "yes", "no", and "unsure". The questions have been widely used both in China and other countries [7, 20]. In the study, we divided the MSM population into three categories according to gender identity, including cisgender MSM (those who agreed with their birth-assigned sex), transgender MSM (those who disagreed with their birth-assigned sex), and gender non-conforming MSM.

\section{Psychosocial characteristics}

The Center for Epidemiological Studies Depression (CES-D) was used to screen the symptoms of depression during the past week [21]. The CES-D has 20 items rated on a 4-point Likert scale ranging from "rarely or none of the time" (0) to "most or all of the time" (3). A higher total score indicates more depressive symptoms. The Chinese version of CES-D has been validated [22, 23] and the Cronbach's alpha was 0.92 in this research.

The Multidimensional Scale of Perceived Social Support (MSPSS) was used to measure social support [24]. The scale consists of three subscales and each subscale includes four items. Response option for each item ranges from 1 (very strongly disagree) to 7 (very strongly agree). A higher total score indicates greater perceived social support. This scale has shown a good validity and reliability in the Chinese population [25]. The Cronbach's alpha was 0.94 in this research. 
The 10-item Connor-Davidson Resilience Scale (CD-RISC10) was used to measure resilience [26]. Responses were rated on a 5-point Likert scale ranging from "not true at all" (0) to "true nearly all the time" (4). A composite score was calculated, with higher total score indicating higher capacity of resilience. This scale has been confirmed validated in Chinese adults $[27,28]$ and the Cronbach's alpha was 0.87 in this research.

Identity concealment was measured by a six-item, 5-point Likert subscale. This subscale was based on nondisclosure subscale developed by Testa [29] and further modified by Outland [30], which was suitable for LGBT population [30]. Items were summed to yield a composite scale score, with a higher score suggesting a higher likelihood of sexual orientation concealment. It has been confirmed validated among Chinese adults [31] and the Cronbach's alpha was 0.91 in this study.

HIV-related sexual behaviors

Information on HIV-related risky sexual behaviors for the past six months was collected, including multiple sexual partners (2 or more were classified as having multiple sexual partners), male partner types (all were regular partners/acquaintances, all were onenight stand/occasional partners, or both regular partners/acquaintances and one-night stand/occasional partners), sex after drugs (yes or no), sex after drinking (yes or no), commercial sexual behavior (yes or no). Participants were also asked to answer frequency of condom use during anal sex in the past six months. The options included "never", "sometimes", "often", "almost always" and "every time". In data analysis, "never" "sometimes", "often", "almost always" were classified as inconsistent condom use.

\subsection{Statistical Analysis}

All analysis of data was conducted using SPSS19.0. The prevalence of gender minorities and $95 \%$ confidence interval $(95 \% \mathrm{Cl})$ were described. The Chi-squared tests and Fisher's exact test were performed to explore the differences of socio-demographic characteristics and HIV-related risky sexual behaviors by gender identity. In terms of psychosocial variables, multiple factor variance analysis was used to explore the relationships between different gender identity groups, which was adjusted for age, monthly income, and sexual orientation based on previous research [32-34]. The Bonferroni correction method was used to conduct a post-hoc analysis. Binary logistic analysis was used to explore how gender identity predicted HIV-related risky sexual behaviors (dichotomous variables, e.g., multiple sexual partners), and the Hosmer-Lemeshow test was used to assess the binary logistic regression. Multinominal logistic analysis was used to assess the relationship between male partner types and gender identity, and -2Log likelihood, Akaike Information Criterion (AIC), Bayesian Information Criterion (BIC) were used to evaluate the model. In both binary logistic regression and multinomial logistic regression, gender identity was coded as independent variable, HIV-related risky sexual behaviors in the past six months were coded as dependent variables, and socio-demographic and psychosocial characteristics as covariates. The level of $\mathrm{P}<0.05$ (two-sides) was set for statistical significance in this study.

\section{Results}

A total of 800 participants were invited into this survey, and 749 participants completed the questionnaire. Participants who did not have not sex with men in the past six months $(n=23)$, and who were not MSM $(n=11)$ were excluded. That left data for 715 MSM eligible for analysis, indicating a response rate of $89.4 \%$. Of the eligible individuals, 502, 106, and 107 participants were recruited from Wuhan, Nanchang, and Changsha, respectively.

As shown in Table 1, the majority of participants were cisgender MSM (91.2\%), transgender and gender non-conforming MSM accounted for $8.8 \%$ (95\% Cl: 6.7\%-10.9\%) of the total MSM population. In all samples, 396 (55.4\%) MSM were between 16 to 25 years of age and $535(74.8 \%)$ had completed college or higher education. 604 (84.5\%) of the participants were unmarried and 111 (15.5\%) were married or divorced. 663(92.7\%) individuals were employed and $362(50.6 \%)$ described that their disposable income exceeded 3000 RMB monthly. 656 (91.7\%) reported that they were homosexual or bisexual, whereas 59(8.3\%) reported as heterosexual or unsure.

After adjusting for age, monthly income, and sexual orientation, gender identity did not significantly predict psychosocial characteristics, except for identity concealment (see Table 2). Specifically, cisgender MSM reported significantly higher scores of identity concealment than transgender (mean difference $=-3.30,95 \% \mathrm{Cl}=-5.86,-0.74, \mathrm{P}=0.01$ ), while the differences among gender non-conforming MSM and the other two groups were not significant. 
Binary logistic regression and the multinominal logistic regression models showed good fit. Compared to cisgender MSM, transgender MSM were more likely to have a one-night stand/occasional partners ( $\mathrm{AOR}=3.49,95 \% \mathrm{Cl}=1.02-11.98$ ), and to have sex after drug use in the past six months ( $A O R=2.57,95 \% \mathrm{Cl}=1.05-6.29)$. No significant differences in HIV-related sexual risky behaviors were observed between gender non-conforming and cisgender MSM.

\section{Discussion}

To our best knowledge, this is the first study to explore gender identity differences in psychosocial characteristics and HIV-related risky sexual behaviors among the MSM subgroup populations in China. In the current study, compared to cisgender MSM, those in the transgender subgroup were more likely to have a one-night stand/occasional partners, to have sex after drug use, and reported a lower likelihood of identity concealment. Consistent with Jobson's research, gender identity played an important role in HIV transmission among the MSM population [7]. Hence, the findings from this study suggest that effective HIV interventions within the MSM population should be separated by the different gender identities.

The study showed that the proportion of transgender and gender non-conforming accounted for $8.8 \%$ of the total MSM population. A study conducted in Peru reported that $23 \%$ of sample were identified as gender minorities among the MSM population [6] , while another study found that $7.2 \%$ of the MSM population were identified as transgender women [35]. This existing discrepancy could be attributed to various assessment tools of gender identify, different cultural norms, or study settings. Though our sample is limited to the recruitment from the central part of China, this study helps to establish the initial epidemic profile of risk factors for transgender and gender non-conforming MSM in China.

Non-significant associations between depression, resilience, perceived social support, and gender identity in the MSM population was consistent with Sandfort's research [36]. The cisgender MSM population were more likely to report high levels of identity concealment than transgender MSM. The lower likelihood of identity concealment among transgender MSM could mean that they have to tolerate more discrimination when compared with the cisgender MSM population [37], which may increase the frequency of condomless anal sex [38] or other HIV-related risk behaviors (e.g. male partner type) [39] and in turn increase their risks of HIV infection. Thus, future research should further explore the relationship between gender identity and psychosocial characteristics within the MSM sub-populations, using additional measures such as discrimination.

Some studies conducted in community population showed that transgender individuals were more likely to have a one-night stand/occasional partners and sex after drug use, when compared to the cisgender MSM population [12, 40]. A study in Brazil reported that transgender individuals engaged in more HIV-related risky sexual behaviors than MSM individuals, including having a higher number of sexual partners, being more likely to engage in sex [41]. Similarly, our study conducted among the MSM population found that compared with cisgender MSM, transgender MSM were more likely to have sex after drug use and have a one-night stand/occasional partners. This is possibly because that transgender MSM have a dual minority identity being both transgender and MSM [42] and due to this dual identity, they perceive stigma when seeking support from service, while the cisgender MSM population do not. The dual minority identity of transgender MSM, as stated, are shown to have a higher level of discrimination and engage in more risky sexual behaviors [7]. Consequently, these results suggested that we should focus on gender minority sub-groups, as transgender MSM have different risks from the larger groups of cisgender MSM.

For gender non-conforming MSM, they may or may not considered themselves to be transgender[43, 44], so they face unique life experiences and may have different psychosocial health outcomes [44]. Halley's research [44] also showed gender non-conforming MSM populations were more likely to have poor mental health and distress in comparison with transgender individuals. However, in this study, gender non-conforming MSM were not found to have significant differences in psychosocial characteristics and HIVrelated risky sexual behaviors in comparison with the cisgender MSM. This is possibly due to small sample size in the study. So, future research needs to increase sample size for further focusing on the population.

Several limitations should be noted in this current study. First, participants were recruited in three Chinese central cities by convenient sampling, and the sample size of transgender MSM and gender non-conforming MSM was small, which impacted the generalizability of the findings. Second, psychosocial outcomes were measured by four self-reported scales, not considering other variables like anxiety, self-esteem, self-efficacy, and copying styles. Also, other HIV-related risk behaviors (PrEP, PEP, substance use) weren't included in the study, and detail information about types of drug or drinking during sex, frequency, duration of use wasn't 
obtained. It is necessary that additional psychosocial and behavioral outcomes are incorporated in further research exploring the relationship between gender identity, psychosocial charactristics, and HIV-related risk behaviors. Third, there was a potential for social desirability bias in the psychosocial characteristic assessment. Fourth, the study ignored the differences within the transgender community, further research is needed to understand the difference between transgender women and transgender men in China. Despite these limitations, this study highlights the prevalence of transgender and gender non-conforming MSM in China, and found gender identity differences in psychosocial characteristics and HIV-related risky sexual behaviors. There is a need for further studies focusing on the association between gender minorities, psychosocial characteristics and HIV related risk behaviors with larger sample and considering more factors.

\section{Conclusions}

In conclusion, the proportion of transgender and non-conforming MSM was 8.8\% in the current study. Compared with the cisgender MSM population, transgender MSM reported low levels of identity concealment, but were more likely to engage in HIV-related risky sexual behaviors, including having a one-night stand/occasional partners and sex after drug use. It is crucial to provide targeted interventions for transgender MSM, a high-risk subgroup. Further research is also needed to focus on the mechanism of the relationships between gender identity, psychosocial characteristics, and HIV-related risky sexual behavior among the MSM subgroups for effectively reducing HIV infection risk.

\section{Declarations}

\section{Acknowledgements}

We would like to thank all participants who generously shared their time and the organizations for their help in data collection.

\section{Authors' contributions}

ZD analyzed this data and wrote manuscript; LW and MG collected this data; $C D$ and DH revised this manuscript; $H Y$ designed this research and revised manuscript; AW and SL edited the language. All authors approved the publication of this manuscript.

\section{Funding}

This study was supported by the National Natural Science Foundation of China (Grant No. 81673196) and the Humanities and Social Science Foundation of Ministry of Education (Grant No. 15YJAZH094). The funder had no role in study design, data collection, analysis, and interpretation of this manuscript.

\section{Availability of data and materials}

Data is available from the corresponding author upon reasonable request.

\section{Ethics approval and consent to participate}

All participants provided written consent and this study was approved by the Medical Ethics Committee at Wuhan University, China.

\section{Consent for publication}

Not applicable

\section{Competing interests}

There is no conflict of interest for all authors.

\section{Abbreviations}

MSM: men who have sex with men; HIV: human immunodeficiency virus; CDC: Centers for Disease Control and Prevention; Cl: confidence interval; AOR: adjust odds ratio; SD: standard deviation; AIC: Akaike Information Criterion; BIC: Bayesian Information 


\section{References}

1. Wallien MS, Cohen-Kettenis PT: Psychosexual outcome of gender-dysphoric children. Journal of the American Academy of Child and Adolescent Psychiatry 2008, 47(12):1413-1423.

2. Lombardi E: Enhancing Transgender Health Care. American journal of public health 2001, 91(6):869-872.

3. Gia, Chodzen, Marco, Hidalgo, Diane, Chen, Robert, Garofalo: Minority Stress Factors Associated With Depression and Anxiety Among Transgender and Gender-Nonconforming Youth. Journal of Adolescent Health Official Publication of the Society for Adolescent Medicine 2018.

4. Bradley, Patrick, White, Holly, Fontenot: Transgender and non-conforming persons' mental healthcare experiences: An integrative review. Archives of Psychiatric Nursing 2019.

5. Beacroft L, Hallett TB: The potential impact of a "curative intervention" for HIV: a modelling study. Global Health Research and Policy 2019, 4(1):18.

6. Trebelcock WL, Lama JR, Duerr A, Sanchez H, Cabello R, Gilada T, Segura P, Reisner SL: HIV pretreatment drug resistance among cisgender MSM and transgender women from Lima, Peru. 2019, 22(11):e25411.

7. Jobson G, Tucker A, de Swardt G, Rebe K, Struthers H, Mclntyre J, Peters R: Gender identity and HIV risk among men who have sex with men in Cape Town, South Africa. AIDS care 2018, 30(11):1421-1425.

8. Lane T, Osmand T, Marr A, Shade SB, Dunkle K, Sandfort T, Struthers H, Kegeles S, Mclntyre JA: The Mpumalanga Men's Study (MPMS): results of a baseline biological and behavioral HIV surveillance survey in two MSM communities in South Africa. PloS one 2014, 9(11):e111063.

9. Sandfort TG, Lane T, Dolezal C, Reddy V: Gender Expression and Risk of HIV Infection Among Black South African Men Who Have Sex with Men. AIDS and behavior 2015, 19(12):2270-2279.

10. Reback CJ, Fletcher JB: HIV prevalence, substance use, and sexual risk behaviors among transgender women recruited through outreach. AIDS and behavior 2014, 18(7):1359-1367.

11. Beyrer C, Baral SD, Walker D, Wirtz AL, Johns B, Sifakis F: The expanding epidemics of HIV type 1 among men who have sex with men in low- and middle-income countries: diversity and consistency. Epidemiologic reviews 2010, 32:137-151.

12. Shan D, Yu M-H, Yang J, Zhuang M-H, Ning Z, Liu H, Liu L, Han M-J, Zhang D-P: Correlates of HIV infection among transgender women in two Chinese cities. Infectious Diseases of Poverty 2018, 007(006):49-58.

13. Peng K, Zhu X, Gillespie A, Wang Y, Gao Y, Xin Y, Qi J, Ou J, Zhong S, Zhao L et al: Self-reported Rates of Abuse, Neglect, and Bullying Experienced by Transgender and Gender-Nonbinary Adolescents in China. JAMA network open 2019, 2(9):e1911058.

14. Sugano E, Nemoto T, Operario D: The impact of exposure to transphobia on HIV risk behavior in a sample of transgendered women of color in San Francisco. AIDS and behavior 2006, 10(2):217-225.

15. Sausa LA, Keatley J, Operario D: Perceived risks and benefits of sex work among transgender women of color in San Francisco. Arch Sex Behav 2007, 36(6):768-777.

16. Maleke K, Makhakhe N, Peters RP, Jobson G, De Swardt G, Daniels J, Lane T, Mclntyre JA, Imrie J, Struthers H: HIV risk and prevention among men who have sex with men in rural South Africa. African journal of AIDS research : AJAR 2017, 16(1):31-38.

17. Zakumumpa H, Bennett S, Ssengooba F: Leveraging the lessons learned from financing HIV programs to advance the universal health coverage (UHC) agenda in the East African Community. Global Health Research and Policy 2019, 4(1):27.

18. Wong NS, Tang W, Han L, Best J, Zhang Y, Huang S, Zheng H, Yang B, Wei C, Pan SW et al: MSM HIV testing following an online testing intervention in China. BMC infectious diseases 2017, 17(1):437.

19. Zhao P, Cao B, Bien-Gund CH, Tang W, Ong JJ, Ding Y, Chen W, Tucker JD, Luo Z: Identifying MSM-competent physicians in China: a national online cross-sectional survey among physicians who see male HIV/STI patients. BMC health services research 2018, 18(1):964.

20. Wang Y, Hu Z, Peng K, Rechdan J, Yang Y, Wu L, Xin Y, Lin J, Duan Z, Zhu X et al: Mapping out a spectrum of the Chinese public's discrimination toward the LGBT community: results from a national survey. 2020, 20(1):669. 
21. Radloff L: The CES-D Scale: A Self-Report Depression Scale for Research in the General Population. Appl Psychol Meas 1977, 1(3):385-401.

22. Wang W, Xiao C, Yao X, Yang Y, Yan H, Li S: Psychosocial health and suicidal ideation among people living with HIV/AIDS: A cross-sectional study in Nanjing, China. 2018, 13(2):e0192940.

23. Jie Z ZW, Fang G, Juan L, Buxin H, Yanzhi C: Development of the Chinese age norms of CES-D in urban area. Chinese Mental Health Journal [Chinese] 2010, 24(2):139-143.

24. Zimet GD, Powell SS, Farley GK, Werkman S, Berkoff KA: Psychometric characteristics of the Multidimensional Scale of Perceived Social Support. Journal of personality assessment 1990, 55(3-4):610-617.

25. Li S, Li L, Zheng H, Wang Y, Zhu X, Yang Y, Yang Y, He J: Relationship between multifaceted body image and negative affect among women undergoing mastectomy for breast cancer: a longitudinal study. Archives of women's mental health 2018, 21(6):681-688.

26. Ehrich J, Mornane A, Powern T: Psychometric Validation of the 10-item Connor-Davidson Resilience Scale. Journal of applied measurement 2017, 18(2):122-136.

27. Ding C, Wang T, Chen X, Li J, Wang W, Huang D, Yan H, Li S: Association of Adverse Childhood Experience and Attention Deficit Hyperactivity Disorder with depressive symptoms among men who have sex with men in China: moderated mediation effect of resilience. $B M C$ public health 2019, 19(1):1706.

28. Zhang M, Zhang J, Zhang F, Zhang L, Feng D: Prevalence of psychological distress and the effects of resilience and perceived social support among Chinese college students: Does gender make a difference?Psychiatry research 2018, 267:409-413.

29. Testa RJ, Habarth J, Peta J, Balsam K, Bockting W: Development of the Gender Minority Stress and Resilience Measure. Psychology of Sexual Orientation \& Gender Diversity 2015, 2(1):65-77.

30. Outland PL: Developing the LGBT minority stress measure. Libraries. Colorado State University; 2016.

31. Ding C, Chen X, Wang W, Yu B, Yang H, Li X, Deng S, Yan H, Li S: Sexual Minority Stigma, Sexual Orientation Concealment, Social Support and Depressive Symptoms Among Men Who have Sex with Men in China: A Moderated Mediation Modeling Analysis. AIDS and behavior 2020, 24(1):8-17.

32. Hu Y, Zhong XN, Peng B, Zhang Y, Liang H, Dai JH, Zhang J, Zhong XH, Huang AL: Comparison of depression and anxiety between HIV-negative men who have sex with men and women (MSMW) and men who have sex with men only (MSMO): a cross-sectional study in Western China. BMJ open 2019, 9(1):e023498.

33. Li D, Li C, Wang Z, Lau JT: Prevalence and associated factors of unprotected anal intercourse with regular male sex partners among HIV negative men who have sex with men in China: a cross-sectional survey. PloS one 2015, 10(3):e0119977.

34. Yang Z, Li S, Zhang R, Na J, Li Y, Mu H, Yu L, Liu L, Sun W, Pan G et al: Current heterosexual marriage is associated with significantly decreased levels of anxiety symptoms among Chinese men who have sex with men. BMC psychiatry 2020, 20(1):151.

35. Soto RJ, Ghee AE, Nunez CA, Mayorga R, Tapia KA, Astete SG, Hughes JP, Buffardi AL, Holte SE, Holmes KK: Sentinel surveillance of sexually transmitted infections/HIV and risk behaviors in vulnerable populations in $\mathbf{5}$ Central American countries. Journal of acquired immune deficiency syndromes (1999) 2007, 46(1):101-111.

36. Sandfort TGM, Bos H, Reddy V: Gender Expression and Mental Health in Black South African Men Who Have Sex with Men: Further Explorations of Unexpected Findings. Arch Sex Behav 2018, 47(8):2481-2490.

37. Feldman J, Romine RS, Bockting WO: HIV Risk Behaviors in the U.S. Transgender Population: Prevalence and Predictors in a Large Internet Sample. J Homosex 2014, 61(11):1558-1588.

38. Poteat T, Ackerman B, Diouf D, Ceesay N, Mothopeng T, Odette KZ, Kouanda S, Ouedraogo HG, Simplice A, Kouame A et al: HIV prevalence and behavioral and psychosocial factors among transgender women and cisgender men who have sex with men in 8 African countries: A cross-sectional analysis. Plos Medicine 2017, 14(11).

39. Kaplan RL, McGowan J, Wagner GJ: HIV prevalence and demographic determinants of condomless receptive anal intercourse among trans feminine individuals in Beirut, Lebanon. Journal of the International AIDS Society 2016, 19(3 Suppl 2):20787.

40. Nemoto T, Luke D, Mamo L, Ching A, Patria J: HIV risk behaviours among male-to-female transgenders in comparison with homosexual or bisexual males and heterosexual females. AIDS care 1999, 11(3):297-312. 
41. Fernandes FR, Zanini PB, Rezende GR, Castro LS, Bandeira LM, Puga MA, Tanaka TS, Castro LS: Syphilis infection, sexual practices and bisexual behaviour among men who have sex with men and transgender women: a cross-sectional study. 2015, 91(2):142-149.

42. Chariyalertsak S, Kosachunhanan N, Saokhieo P, Songsupa R, Wongthanee A, Chariyalertsak C, Visarutratana S, Beyrer C: HIV incidence, risk factors, and motivation for biomedical intervention among gay, bisexual men, and transgender persons in Northern Thailand. PloS one 2011, 6(9):e24295.

43. Steensma TD, Kreukels BP, de Vries AL, Cohen-Kettenis PT: Gender identity development in adolescence. Hormones and behavior 2013, 64(2):288-297.

44. Crissman HP, Stroumsa D, Kobernik EK, Berger MB: Gender and Frequent Mental Distress: Comparing Transgender and Non-

Transgender Individuals' Self-Rated Mental Health. Journal of women's health (2002) 2019, 28(2):143-151.

\section{Tables}

Table 1. Socio-demographic characteristics by gender identity, n (\%).

\begin{tabular}{|c|c|c|c|c|c|c|}
\hline Variables & Total & Cisgender MSM & Gender non-conforming & Transgender & $\chi^{2}$ & $\mathrm{P}$ \\
\hline Total & & $652(91.2)$ & $37(5.2)$ & $26(3.6)$ & & \\
\hline Age group (in years) ${ }^{a}$ & & & & & - & 0.95 \\
\hline $16-25$ & $396(55.4)$ & $360(55.2)$ & $22(59.5)$ & $14(53.8)$ & & \\
\hline $26-35$ & $211(29.5)$ & 193(29.6) & $10(27.0)$ & $8(30.8)$ & & \\
\hline $36-45$ & $69(9.7)$ & $64(9.8)$ & $2(5.4)$ & $3(11.5)$ & & \\
\hline $45-59$ & $39(5.5)$ & $35(5.4)$ & $3(8.1)$ & $1(3.8)$ & & \\
\hline Ethnicity ${ }^{\text {a }}$ & & & & & - & 0.29 \\
\hline Han group & $677(94.7)$ & $618(94.8)$ & $36(97.3)$ & $23(88.5)$ & & \\
\hline Others & $38(5.3)$ & $34(5.2)$ & $1(2.7)$ & $3(11.5)$ & & \\
\hline Educational level & & & & & 6.54 & 0.04 \\
\hline High school or lower & $180(25.2)$ & $156(23.9)$ & $13(35.1)$ & $11(42.3)$ & & \\
\hline College or higher & $535(74.8)$ & $496(76.1)$ & $24(64.9)$ & $15(57.7)$ & & \\
\hline Marital status & & & & & 0.66 & 0.73 \\
\hline Unmarried & $604(84.5)$ & $553(84.8)$ & $30(81.0)$ & $21(80.8)$ & & \\
\hline Married/divorced & $111(15.5)$ & $99(15.2)$ & 7(18.9) & $5(19.2)$ & & \\
\hline Employment status ${ }^{a}$ & & & & & - & 0.62 \\
\hline Employed & $663(92.7)$ & $605(92.8)$ & $35(94.6)$ & $23(88.5)$ & & \\
\hline Unemployed & $52(7.3)$ & $47(7.2)$ & $2(5.4)$ & $3(11.5)$ & & \\
\hline Monthly income (RMB) & & & & & 10.18 & 0.12 \\
\hline$<1000$ & $91(12.7)$ & $80(12.3)$ & $6(16.2)$ & $5(19.2)$ & & \\
\hline $1000-3000$ & $262(36.6)$ & $242(37.1)$ & $10(27.0)$ & $10(38.5)$ & & \\
\hline $3001-6000$ & $229(32.0)$ & $215(33.0)$ & $8(21.6)$ & $6(23.1)$ & & \\
\hline$>6000$ & 133(18.6) & $115(17.6)$ & $13(35.1)$ & $5(19.2)$ & & \\
\hline Sexual orientation & & & & & 67.80 & $<0.001$ \\
\hline Homosexual & $520(72.7)$ & $495(75.9)$ & $11(29.7)$ & $14(53.8)$ & & \\
\hline Bisexual & $136(19.0)$ & 116(17.8) & $11(29.7)$ & $9(34.6)$ & & \\
\hline Unsure/heterosexual & $59(8.3)$ & $41(6.3)$ & $15(40.5)$ & $3(11.5)$ & & \\
\hline
\end{tabular}




\begin{tabular}{|c|c|c|c|c|c|c|}
\hline Variables $^{\mathrm{a}}$ & Total & Cisgender & $\begin{array}{l}\text { Gender non- } \\
\text { conforming }\end{array}$ & Transgender & $\mathrm{F}$ & $\mathrm{P}$ \\
\hline Depression & $17.66 \pm 10.50(0,58)$ & $17.52 \pm 10.46(0,58)$ & $19.54 \pm 11.27(2,48)$ & $18.58 \pm 10.43(2,44)$ & 0.42 & 0.65 \\
\hline $\begin{array}{l}\text { Perceived } \\
\text { social } \\
\text { support }\end{array}$ & $60.42 \pm 12.55(12,84)$ & $60.57 \pm 12.38(12,84)$ & $60.46 \pm 14.89(25,84)$ & $56.46 \pm 13.03(25,74)$ & 1.14 & 0.32 \\
\hline Resilience & $36.70 \pm 8.48(10,50)$ & $36.75 \pm 8.51(10,50)$ & $36.14 \pm 8.45(10,50)$ & $36.38 \pm 8.11(17,50)$ & 0.08 & 0.92 \\
\hline $\begin{array}{l}\text { Identity } \\
\text { concealment } \\
\text { b }\end{array}$ & $17.05 \pm 6.63(6,30)$ & $17.19 \pm 6.61(6,30)$ & $16.68 \pm 6.15(6,30)$ & $14.00 \pm 7.07(6,29)$ & 3.33 & 0.04 \\
\hline
\end{tabular}

Table 2. Psychosocial characteristics by gender identity, Mean \pm SD (min-max).

Note: SD standard deviation

a: adjust for age, monthly income and sexual orientation.

b: cisgender VS gender non-confirming: mean difference $=-0.75$ (95\%CI $=-2.98,1.48), \mathrm{P}=0.51$; cisgender VS transgender: mean difference $=-3.30(95 \% \mathrm{CI}=-5.86,-0.74), \mathrm{P}=0.01$ and gender non-confirming VS transgender: mean difference $=-2.55(95 \% \mathrm{CI}=-0.73$, 5.83), $\mathrm{P}=0.13$.

Table 3. HIV-related risky behaviors by gender identity, n (\%). 


\begin{tabular}{|c|c|c|c|c|c|c|}
\hline Variables & Total & $\begin{array}{l}\text { Cisgender } \\
\text { MSM }\end{array}$ & $\begin{array}{l}\text { Gender non- } \\
\text { Conforming }\end{array}$ & Transgender & $\chi^{2}$ & $\mathrm{P}$ \\
\hline Multiple sexual partners & & & & & 0.69 & 0.71 \\
\hline Yes & $381(53.3)$ & $306(46.9)$ & $15(40.5)$ & $13(50.0)$ & & \\
\hline No & $334(46.7)$ & $346(53.1)$ & $22(59.5)$ & $13(50.0)$ & & \\
\hline Male partner types ${ }^{a}$ & & & & & - & 0.08 \\
\hline All were regular partners/acquaintances & $335(53.5)$ & $313(54.7)$ & $16(48.5)$ & $6(28.6)$ & & \\
\hline $\begin{array}{l}\text { All were one -night stand/occasional } \\
\text { partners }\end{array}$ & $79(12.6)$ & $68(11.9)$ & $5(15.2)$ & $6(28.6)$ & & \\
\hline $\begin{array}{l}\text { Both regular partners/acquaintances and } \\
\text { one-night stand/occasional partners }\end{array}$ & 212(33.9) & $191(33.4)$ & $12(36.4)$ & $9(42.9)$ & & \\
\hline Sex after drugs & & & & & 6.32 & 0.04 \\
\hline Yes & $100(14.0)$ & $87(13.3)$ & $5(13.5)$ & $8(30.8)$ & & \\
\hline No & $615(86.0)$ & $565(86.7)$ & $32(86.5)$ & 18(69.2) & & \\
\hline Sex after drinking & & & & & 0.85 & 0.65 \\
\hline Yes & $281(39.3)$ & $258(39.6)$ & $12(32.4)$ & $11(42.3)$ & & \\
\hline No & $434(60.7)$ & $394(60.4)$ & $30(81.0)$ & $21(80.8)$ & & \\
\hline Commercial sexual behavior $^{a}$ & & & & & - & 0.049 \\
\hline Yes & $12(1.9)$ & $9(1.6)$ & $1(3.0)$ & $2(9.5)$ & & \\
\hline No & $614(98.1)$ & $563(98.4)$ & $32(97.0)$ & $19(90.5)$ & & \\
\hline Inconsistent condom use & & & & & 1.81 & 0.41 \\
\hline Yes & $286(49.2)$ & $257(48.4)$ & $18(60.0)$ & $11(55.0)$ & & \\
\hline No & $295(50.8)$ & 274(51.6) & $12(40.0)$ & $9(45.0)$ & & \\
\hline
\end{tabular}

Note: a: Fisher's exact test

Table 4. The relationship between gender identity and HIV-related risky sexual behaviors (Reference Category $=$ cisgender MSM). 


\begin{tabular}{|c|c|c|c|c|c|c|c|c|}
\hline \multirow[t]{2}{*}{ Variables a b } & \multicolumn{4}{|c|}{ Gender non-conforming } & \multicolumn{4}{|c|}{ Transgender } \\
\hline & $\bar{B}$ & $\bar{P}$ & AOR & $95 \% \mathrm{CI}$ & $\bar{B}$ & $\mathrm{P}$ & AOR & $95 \%$ CI \\
\hline iple sexual partners (yes) & -0.13 & 0.73 & 0.88 & $0.43-1.80$ & 0.12 & 0.77 & 1.13 & $0.51-2.52$ \\
\hline \multicolumn{9}{|l|}{ partner type } \\
\hline $\begin{array}{l}\text { ll were regular } \\
\text { ters/acquaintances }\end{array}$ & & & ref & & \multicolumn{4}{|c|}{ ref } \\
\hline $\begin{array}{l}\text { ll were one-night } \\
\text { d/occasional partners }\end{array}$ & 0.16 & 0.78 & 1.17 & $0.38-3.64$ & 1.25 & 0.047 & 3.49 & $1.02-11.98$ \\
\hline $\begin{array}{l}\text { oth } \\
\text { lar partners/acquaintances } \\
\text { one-night stand/occasional } \\
\text { cers }\end{array}$ & 0.12 & 0.78 & 1.12 & $0.49-2.56$ & 0.73 & 0.18 & 2.08 & $0.71-6.09$ \\
\hline after drugs (yes) & -0.17 & 0.75 & 0.84 & $0.30-2.39$ & 0.94 & 0.04 & 2.57 & $1.05-6.29$ \\
\hline after drinking (yes) & -0.49 & 0.20 & 0.61 & $0.29-1.30$ & -0.04 & 0.93 & 0.96 & $0.43-2.18$ \\
\hline mercial sexual behavior & 0.55 & 0.65 & 1.73 & $0.17-18.00$ & 1.71 & 0.06 & 5.51 & $0.91-33.39$ \\
\hline nsistent condom use (yes) & 0.58 & 0.16 & 1.79 & $0.79-4.08$ & 0.08 & 0.86 & 1.09 & $0.43-2.78$ \\
\hline
\end{tabular}

Note: AOR: adjust odds ratio; CI: confidence interval.

a: All models controlled for age, ethnicity, educational level, marital status, employment status, monthly income, sexual orientation, depression, perceived social support, resilience and identity concealment.

b: Hosmer and Lemeshow Test: multiple sexual partners, Chi-square $=7.69, \mathrm{df}=8, \mathrm{P}=0.47$; sex after drug, Chi-square $=7.26$, $\mathrm{df}=8, \mathrm{P}=0.51$; sex after drinking, Chi-square $=4.85, \mathrm{df}=8, \mathrm{P}=0.77$; commercial sexual behaviors, Chi-square $=2.24, \mathrm{df}=8, \mathrm{P}=0.97$; inconsistent condom use, Chi-square $=2.52, \mathrm{df}=8, \mathrm{P}=0.96$. Model fit of male partner types: $-2 \mathrm{Log}$ likelihood $=1156.90$, $\mathrm{AIC}=1216.90$, $\mathrm{BIC}=1350.08$.

\section{Figures}




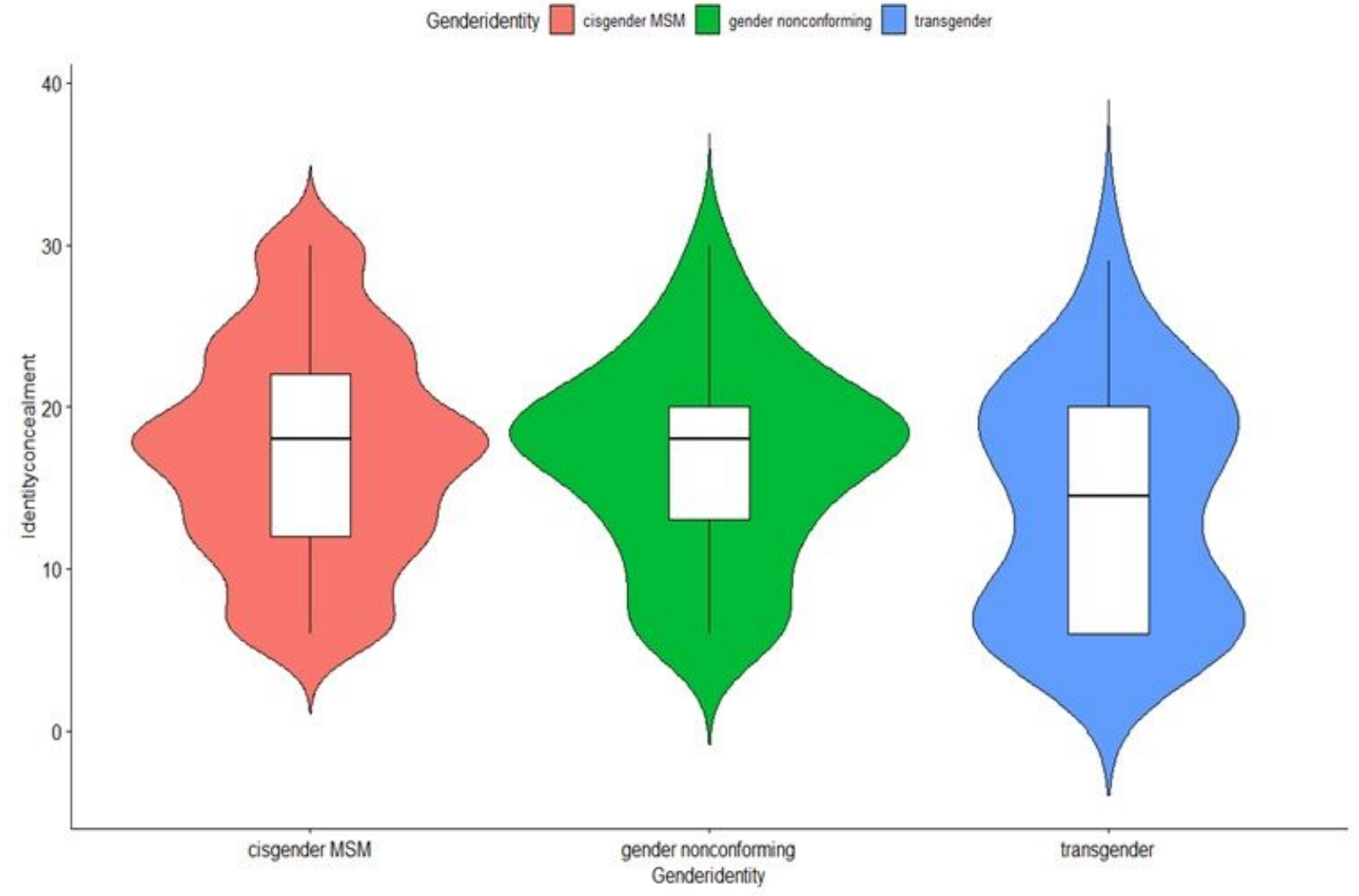

\section{Figure 1}

The Identity concealment characteristics separated by gender identity. Note: *:p<0.05 